\title{
Falha por Stress Cracking em Híbridos PET/Argila
}

\author{
Edvânia Trajano Teófilo, Emanuela Santos Silva, Suédina Maria Lima Silva, Marcelo Silveira Rabello \\ Unidade Acadêmica de Engenharia de Materiais, Universidade Federal de Campina Grande - UFCG
}

\begin{abstract}
Resumo: O presente estudo investiga a falha por stress cracking em polímeros contendo argila montmorilonítica. Ensaios de tração e de relaxação de tensão foram conduzidos para avaliar a resistência ao stress cracking do PET puro e dos híbridos PET/argila em contato com soluções aquosas de hidróxido de sódio. As análises por difratometria de raios-X evidenciaram que a argila adicionada não gerou uma estrutura esfoliada e sim a obtenção de microcompósitos. Os resultados mostraram que a presença de argila dispersa no PET causa concentração de tensão, o que exerce forte influência no comportamento de stress cracking, com os efeitos sendo afetados pela ordenação lamelar e ao teor de carga, resultando em maiores taxas de relaxação de tensão. A argila com menor regularidade no empilhamento lamelar, embora ocasione menor concentração de tensão, favorece mais o fissuramento superficial da matriz, sugerindo que o efeito de barreira ao fluido não foi efetivo. Medidas de massa molar evidenciaram que a argila acelera o ataque químico da matriz quando elevadas concentrações de solução são utilizadas, porém diminui o efeito da tensão mecânica na degradação.
\end{abstract}

Palavras-chave: PET, argila, stress cracking, nanocompósitos.

\section{Stress Cracking Failure of PET/Clay Composites}

Abstract: This study investigates stress-cracking failure of polymer/clay composites. Tensile and stress relaxation tests were conducted to evaluate the stress cracking resistance of PET and PET/clay in the presence of sodium hydroxide aqueous solution. The X-ray diffraction analyses showed that the clay formed a typical structure of a microcomposite, and not an exfoliated structure. The presence of clay causes stress concentration, with a strong consequence to the stress cracking behavior, but the effects depend on the lamellar ordering and the content of clay. When the clay lamellae are less ordered the rise in stress concentration is lower, but causes more surface cracks on the polymer, suggesting that the barrier effect was not very effective. Molar mass measurements showed that the clay accelerates the chemical attack of the matrix when higher concentrations of $\mathrm{NaOH}$ are used, but reduces the effect of mechanical stress on degradation.

Keywords: PET, clay, stress-cracking, nanocomposites.

\section{Introdução}

O poli (tereftalato de etileno) (PET) é um dos mais importantes plásticos de engenharia da atualidade, sendo um dos termoplásticos mais produzidos no mundo ${ }^{[1]}$. Apresenta uma excelente combinação de rigidez e tenacidade, alta resistência ao calor, estabilidade dimensional, capacidade de isolamento elétrico, além da possibilidade de se apresentar no estado amorfo (transparente), parcialmente cristalino e orientado (translúcido) e altamente cristalino (opaco) ${ }^{[2]}$. Entretanto, desvantagens tais como baixa taxa de cristalização e baixa temperatura de distorção térmica têm limitado suas aplicações como um plástico de engenharia no estado amorfo $^{[3]}$. Alguns autores defendem a incorporação de argilas, produzindo nanocompósitos, como uma maneira de melhorar as propriedades do PET $^{[4-6]}$, argumentando que podem ser conseguidos aumentos consideráveis na temperatura de cristalização, temperatura de distorção térmica e no módulo de elasticidade com a preparação de híbridos PET/argila, o que resultaria na ampliação do seu campo de aplicação ${ }^{[7]}$. O bom desempenho durante o uso, entretanto, também está fortemente associado às condições ambientais em que o artefato está submetido, o que inclui: temperatura, presença de umidade, agentes químicos, radiação solar, etc., as quais podem ser imprevisíveis e muitas vezes subestimadas. Dentre esses vários fatores ambientais que podem contribuir para a redução da durabilidade do PET e da maioria dos polímeros comerciais, tem-se o environmental stress cracking - $\mathrm{ESC}^{[8]}$.

O fenômeno de ESC tem sido reportado como responsável pela maioria dos casos de falha prematura em produtos plásticos, ocorrendo desde fissuramentos superficiais à fratura frágil do produto, mesmo em polímeros considerados dúcteis. Howard, pesquisador pioneiro do ESC, em 1959 definiu o fenômeno como uma falha de natureza frágil iniciada na superfície de uma amostra ou peça de PE (hoje se abrange para todos os materiais poliméricos) sujeita a tensões poliaxiais e em contato com um meio (fluido agressivo) em que estando ausente a falha não ocorreria sob as mesmas condições de tensão ${ }^{[9]}$. Acredita-se que o fluido agressivo atue localmente, causando plastificação, que juntamente com a tensão mecânica, facilita o deslocamento molecular e a formação de microfibrilas ${ }^{[10,11]}$. Essa ação, portanto, depende do nível de interação entre o polímero e o fluido. Uma investigação detalhada sobre o tipo de produtos químicos que causam stress cracking em polímeros foi realizado por Hansen e colaboradores ${ }^{[12-14]}$, mostrando que os fluidos que atuam como agentes de ESC têm

Autor para correspondência: Marcelo Silveira Rabello, Unidade Acadêmica de Engenharia de Materiais, Universidade Federal de Campina Grande - UFCG, Rua Aprigio Veloso, 882, CEP 58109-900, Campina Grande, PB, Brasil, e-mail: marcelo@dema.ufcg.edu.br 
interação intermediária com o polímero em comparação com um bom solvente e com um não solvente. Exemplos de fluidos agressivos, a depender do tipo de polímero, incluem líquidos orgânicos (solventes), óleos sintéticos e naturais, detergentes, agentes desmoldantes, plastificantes, formulações de adesivos, tintas, lubrificantes e, em casos extremos, os óleos que ocorrem naturalmente do contato da impressão digital[ ${ }^{[15]}$.

No que se refere a polímeros contendo argilas, não se localizou nenhuma menção na literatura especializada tratando do efeito desse tipo de carga na falha por stress cracking, de forma que o presente estudo pode ser considerado como pioneiro no assunto. Sabe-se que em sistemas intercalados e/ou delaminados tem-se uma redução nos fenômenos difusionais, o que poderia dificultar a penetração do fluido agressivo, o que retardaria a falha por stress cracking. Por outro lado, a presença de argila pode provocar concentração de tensão localizada, promovendo um aumento na tendência ao stress cracking. Assim, a influência desse tipo de carga precisa ser investigada, sendo este o principal objetivo do presente estudo.

Dependendo do tipo de dispersão das lamelas de argila no polímero três tipos de híbridos podem ser obtidos: imiscível, intercalado ou esfoliado. O caso ideal, mas de difícil obtenção, é o esfoliado, no qual as lamelas de argila estão dispersas homogeneamente na matriz polimérica em forma de folhas individuais em que a ordem de empilhamento original é completamente perdida. Um híbrido intercalado é obtido quando ocorre a inserção de poucas moléculas poliméricas entre as camadas do argilomineral, aumentando a distância interplanar basal sem destruir seu empilhamento natural. Já em sistemas imiscíveis, também conhecidos como microcompósitos, as moléculas do polímero não intercalam a argila, e esta atua como uma carga em um compósito convencional, ou seja, a estrutura contém tactoids de argila com camadas agregadas ${ }^{[16,17]}$, sendo mais propensa, portanto, a concentrar tensão. A concentração de tensão pode ser altamente indesejável em situações de exposição do híbrido a fluidos agressivos, caso as propriedades de barreira proporcionadas pela nanocarga não sejam efetivas.

O objetivo do presente estudo é avaliar o comportamento de stress cracking de microcompósitos PET-montmorilonita em contato com soluções aquosas de hidróxico de sódio. Uma vez que o efeito da concentração de tensão promovida pela argila pode ser mais evidente em microcompósitos, esse tipo de híbrido foi escolhido.

\section{Experimental}

Utilizou-se nesse estudo o PET codificado como Cleartuf Turbo (viscosidade intrínseca $0,75 \mathrm{dl} / \mathrm{g}$ ), fornecido pela M\&G Polímeros do Brasil, e como carga uma argila montmorilonítica comercial purificada (Cloisite $\mathrm{Na}^{+}$), proveniente da Southern Clay Products, Texas/EUA. Para a preparação dos corpos de prova, o polímero e a argila, previamente secos (polímero: $160^{\circ} \mathrm{C} / 6 \mathrm{~h}$; argila: $60^{\circ} \mathrm{C} / 6 \mathrm{~h}$ e $110^{\circ} \mathrm{C} / 24 \mathrm{~h}$ ), foram misturados na proporção de $6: 1 \mathrm{em}$ um reômetro de torque Haake. O concentrado obtido foi então misturado ao PET puro em uma extrusora dupla rosca corrotacional interpenetrante, em quantidade que resultasse em híbridos com teores nominais de 1,3 e 5\% em massa de argila. Os híbridos PET/argila e o PET puro foram secos $\left(160^{\circ} \mathrm{C}\right.$ por 6 horas $)$ e moldados na forma de corpos de prova de tração tipo I (ASTM D-638) em uma injetora Fluidmec operando a $260^{\circ} \mathrm{C}$ e com temperatura do molde de $8^{\circ} \mathrm{C}$. Nessas condições, o PET produzido não desenvolve cristalinidade, sendo essencialmente amorfo.

As análises de difração de raios $\mathrm{X}$ nos corpos de prova injetados foram conduzidas em um equipamento Shimadzu XDR-6000 a 40kV e 30mA e com um comprimento de onda $\lambda \mathrm{CuK} \alpha=1,5418 \AA$ utilizando um intervalo de $2 \theta$ entre $2 \mathrm{e}$ $12^{\circ}$ a uma taxa de $2 \%$ min.

Os testes de resistência ao stress cracking no PET puro e nos híbridos PET/argila foram conduzidos em uma máquina universal de ensaio DL da EMIC em duas condições diferentes: (i) sob relaxação de tensão nas cargas de 1300 e $1600 \mathrm{~N}$ (i.e., sob deformação constante) e (ii) durante ensaio de tração convencional. Como fluido agressivo foram utilizadas soluções aquosas de hidróxido de sódio $(\mathrm{NaOH})$ nas concentrações de 0,25; 1 e 3M. Uma vez que foi verificado que a quantidade de solução exerce forte influencia na falha por ESC ${ }^{[18]}$, as soluções foram aplicadas na superfície dos corpos de prova em quantidade controlada ( 5 gotas - o que corresponde a cerca de $0,25 \mathrm{ml}$ ) com o auxílio de um papel filtro (tamanho $5 \times 1 \mathrm{~cm}$ e espessura $205 \mathrm{~mm}$ ). As soluções foram gotejadas no papel filtro e então aderidas à superfície do corpo de prova, de forma que o líquido ficasse em contato com o polímero durante todo o período de teste (tração ou relaxação). Tomou-se o cuidado de utilizar sempre o mesmo conta-gotas e um papel novo a cada aplicação.

Um estudo de absorção de $\mathrm{NaOH}$ pelo PET foi realizado através medidas da variação de massa dos corpos de prova em função do tempo de submersão utilizando uma balança analítica (Quimes) com precisão de 0,0001g. Realizou-se também medidas da viscosidade intrínseca de acordo com o método ASTM D 4603. A viscosidade intrínseca $[\eta]$ foi calculada a partir de uma única medida da viscosidade relativa e a massa molar ponderal média $\left(\mathrm{M}_{\mathrm{w}}\right)$ foi determinada pela equação abaixo ${ }^{[19]}$.

$$
\overline{M_{w}}=\sqrt[0,68]{\frac{[\eta]}{4,68 \times 10^{-4}}}
$$

Após os ensaios de ESC, foram feitas análises visuais das superfícies dos corpos de prova, as quais foram registradas em fotomacrografias digitais. Nesta análise utilizou-se uma câmera Nikon D40 com resolução de 300 dpi e comprimento focal mínimo de $31 \mathrm{~mm}$. As superfícies de fratura foram inspecionadas em um microscópio eletrônico de varredura Shimadzu SSX 550 operando a $10 \mathrm{kV}$, após aplicação de uma cobertura de ouro.

\section{Resultados e Discussão}

Pela monitoração da presença, forma, intensidade e posição dos picos de DRX é possível fazer uma atribuição preliminar sobre a estrutura ou morfologia de nanocompósitos ${ }^{[20]}$. Em um difratograma de raios-X o pico de interesse na caracterização de híbridos polímero/argila, que indica o espaçamento basal $\left(\mathrm{d}_{001}\right)$ da argila, está entre $2^{\circ}$ 
e $10^{\circ[21]}$. O completo desaparecimento desse pico indica o estado de esfoliação. O deslocamento desse pico para um $2 \theta$ menor indica o estado de intercalação. O não deslocamento desse pico ou o deslocamento para um $2 \theta$ maior, indica o estado de agregação das lamelas de argila na forma não intercalada ${ }^{[16]}$. Os difratogramas de raios-X da argila Cloisite $\mathrm{Na}^{+}$e dos híbridos PET/argila estão mostrados na Figura 1, com marcação da localização do pico de interesse. Fica evidenciada a obtenção de microcompósitos tanto para o híbrido obtido com a argila seca a $60^{\circ} \mathrm{C}$ por $6 \mathrm{~h}$ (codificado como H1) quanto para os híbridos obtidos com a argila seca a $110^{\circ} \mathrm{C}$ por $24 \mathrm{~h}$ (codificados como $\mathrm{H} 2$ ), uma vez que o pico referente à reflexão dos planos $\mathrm{D}_{001}$ da argila não foi alterado nos compósitos (Figura 1a).

A obtenção de microcompósito pode ter sido favorecida pelo fato de se ter utilizado uma argila natural, com pequena distância interplanar basal, o que dificultou a penetração das cadeias poliméricas entre as suas camadas. Os oxigênios presentes na superfície externa do argilomineral são compatíveis com polímeros contendo grupos funcionais polares ${ }^{[4]}$, como o PET, e assim pode haver interações com a argila mesmo em seu estado natural, conforme observado por Calcagno et al. ${ }^{[22]}$ que relatam a obtenção de morfologia esfoliada ao utilizar montmorilonita sódica (Cloisite $\mathrm{Na}^{+}$) em matriz de PET. Segundo os autores, a interação polímero-argila e as condições de processamento podem ser fatores suficientes para quebrar a organização lamelar e dispersar a argila na matriz polimérica. No presente estudo, assim como em outros da literatura ${ }^{[23-25]}$, as condições utilizadas não foram suficientes para obter tal feito e, portanto, estruturas típicas de microcompósitos foram obtidas, mesmo nos casos em que se utilizou argila organofílica para favorecer a intercalação do polímero[ ${ }^{[24,25]}$.

\section{Influência da morfologia}

Os resultados a seguir evidenciam que o tempo e temperatura de secagem da argila podem interferir de forma significativa na sua interação com o polímero e, portanto, na estrutura de empilhamento das lamelas de argila. Para os híbridos com $1 \%$ de argila observa-se intensidade de picos de difração diferentes conforme o tipo de secagem (ver Figura 1b). Isso é um indicativo de diferenças morfológicas entre eles, uma vez que a intensidade do pico, considerando a mesma quantidade de carga, está relacionada com a regularidade do empilhamento das lamelas de $\operatorname{argila}^{[20]}$. Quando se trata de teores diferentes, quanto maior o teor de carga mais definido o pico de difração (conforme observado para os híbridos com 3 e $5 \%$ de argila na Figura 1b). Assim, pode-se considerar que o híbrido $\mathrm{H} 2-1 \%$, por apresentar intensidade de pico menor que o híbrido $\mathrm{H} 1-1 \%$, possivelmente apresente lamelas de argila com empilhamento mais desordenado, o que pode conferir maior área de contato com a matriz polimérica. Tal suposição é reforçada pelas diferenças apresentadas entre as propriedades mecânicas de ambos os materiais (mostradas adiante na Figura 2).

A Figura 2 mostra a influência da morfologia nas propriedades mecânicas dos híbridos como também na resistência ao stress cracking desses materiais em contato com solução aquosa de $\mathrm{NaOH}$ na concentração de $1 \mathrm{M}$. Observa-se que a incorporação de $1 \%$ de argila diminui em cerca de $10 \%$ a resistência do PET, tanto na ausência como na presença do fluido agressivo. Os efeitos da ação combinada da tensão mecânica e fluido agressivo é a perda notável de propriedades mecânicas. Na presença da solução aquosa, tanto o PET quanto os híbridos PET/ argila apresentaram falha catastrófica com pequenos níveis de deformação, sendo a diminuição nos valores de resistência mecânica de aproximadamente $40 \%$ e $50 \%$ para o PET e os híbridos, respectivamente (Figura 2a). Já em relação à capacidade de deformação do PET, observa-se que a incorporação de argila fragiliza o material e que essa fragilização varia com a morfologia obtida (Figura 2b). A deformação máxima do PET em estudo é superior a $450 \%$, diminuindo para aproximadamente $160 \%$ quando se incorpora argila e esta mantem um empilhamento ordenado de suas lamelas na forma agregada (híbrido H1-1\%), caindo ainda mais esse valor para $30 \%$ quando se consegue certa desordem no empilhamento lamelar (híbrido H2-1\%). De acordo com Carastan ${ }^{[26]}$, a presença de cargas microscópicas geralmente aumenta a rigidez do polímero através da redução da mobilidade das cadeias poliméricas.

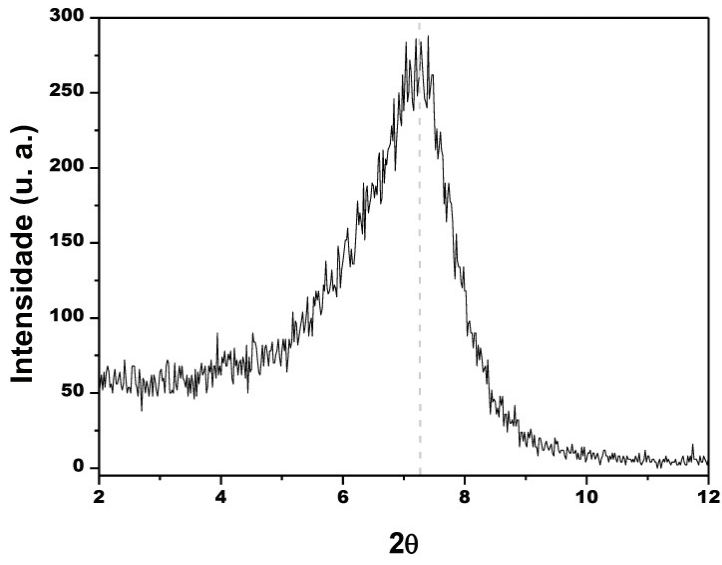

(a)

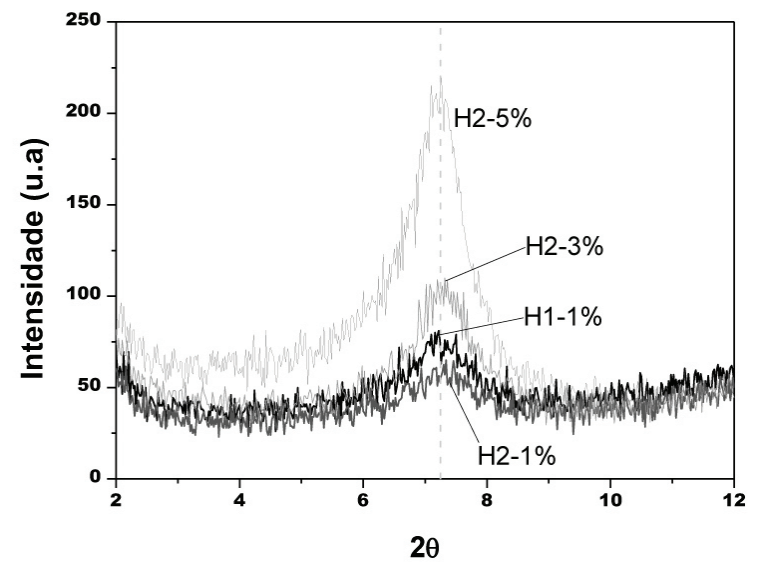

(b)

Figura 1. Difratogramas de DRX da argila pura (a) e dos híbridos PET/argila com teores de 1, 3 e 5\% (b). 
O híbrido $\mathrm{H} 2-1 \%$ apresentou ductilidade inferior ao híbrido $\mathrm{H} 1-1 \%$, considerando que possua cargas lamelares mais desordenadas e apresenta maior área de contato carga/ matriz, resultando em uma maior imobilização ocasionada pela argila. Dessa forma os dados de propriedades mecânicas estão consistentes com os de DRX.

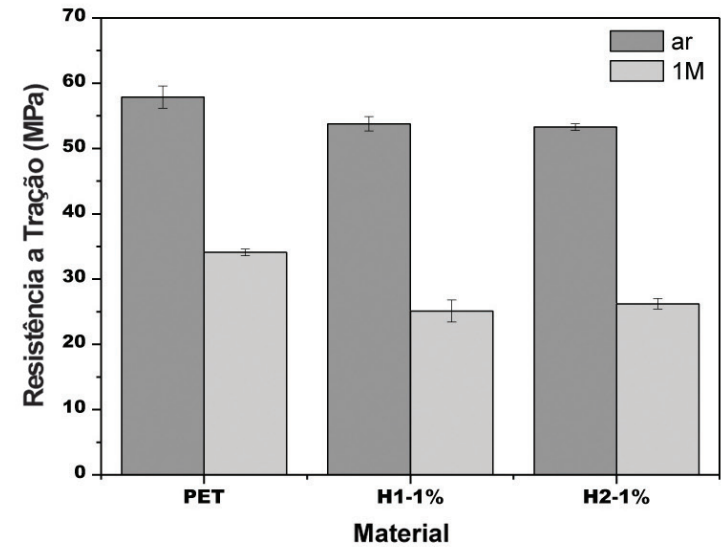

(a)
A Figura 3 mostra imagens de microscopia eletrônica de varredura das superfícies de fratura do PET e do híbrido H1-1\% em contato com solução aquosa de $\mathrm{NaOH}$ na concentração de $1 \mathrm{M}$. Observa-se que a falha teve início na superfície de contato com a solução, evidenciada pela região espelho, e que o padrão de propagação da trinca

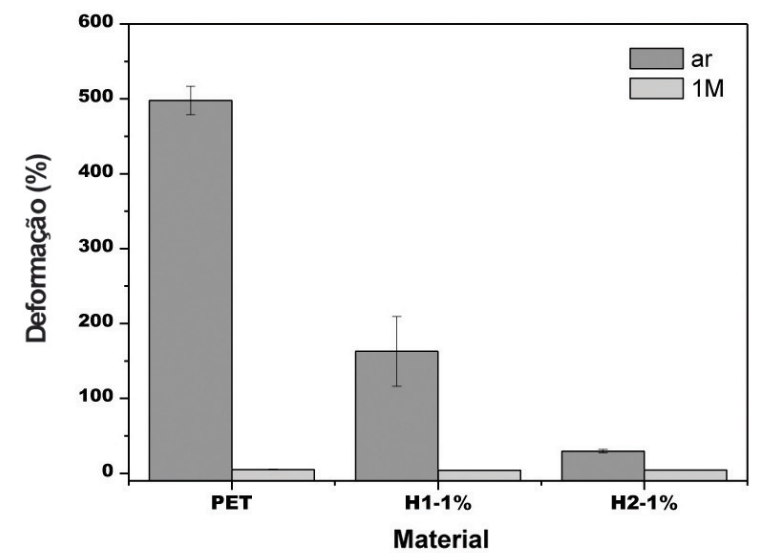

(b)

Figura 2. Propriedades mecânicas do PET e dos híbridos PET/argila na ausência (ar) e na presença de solução aquosa de $\mathrm{NaOH}$ na concentração de $1 \mathrm{M}$.

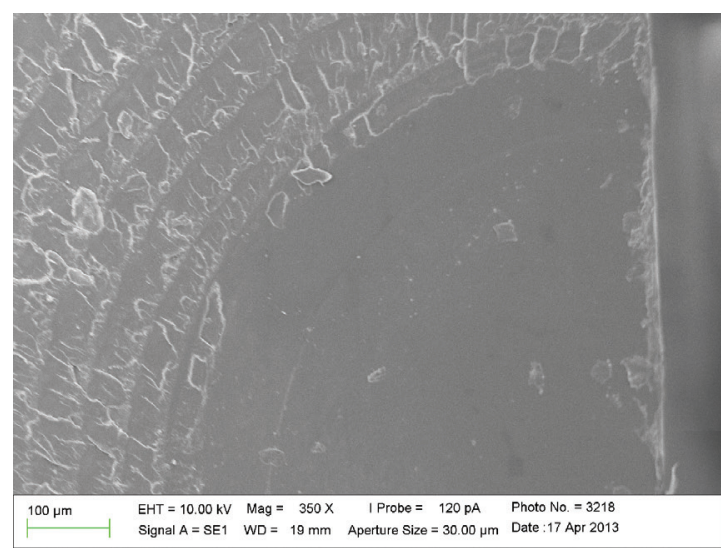

(a)

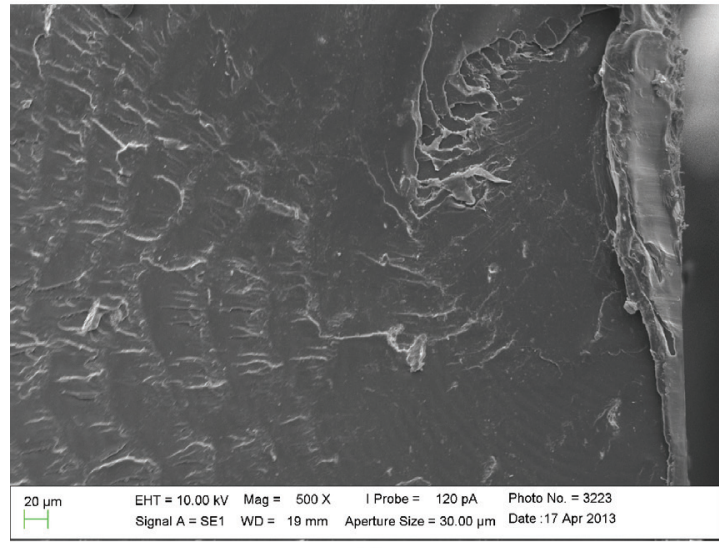

(c)

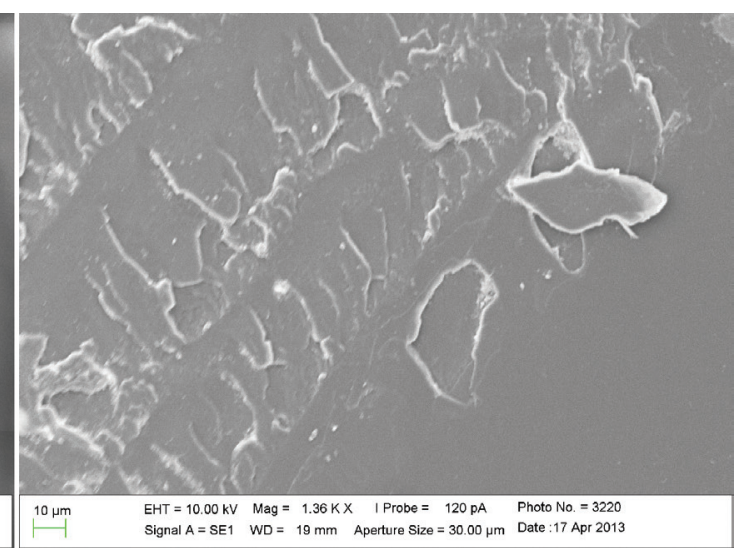

(b)

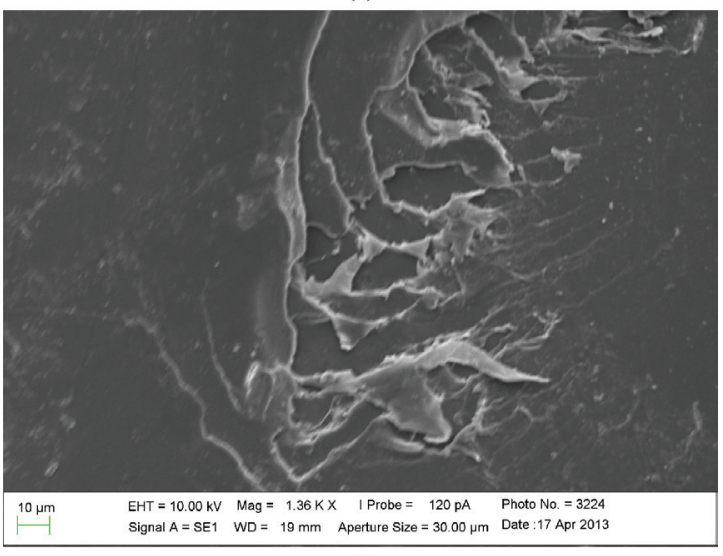

(d)

Figura 3. Micrografias da superfície de fratura de corpos de prova de PET e do híbrido com 1\% de argila ensaiados sob tração e na presença de solução aquosa de $\mathrm{NaOH}$ na concentração de 1M. (a) PET; (b) ampliação de "a"; (c) híbrido H1-1\%; (d) ampliação de "c". As setas indicam o lado em contato com a solução, que coincide com o início da fratura. 
é semelhante em ambos os materiais. Esse padrão é caracterizado por uma região lisa, mostrando o início da trinca em propagação lenta, seguida de uma região rugosa e ondulada, mostrando que a trinca atinge o tamanho crítico e se propaga com padrão frágil, pois avança em zonas formando marcas de fratura semelhantes às marcas de praia. Isso sugere que a fratura pode estar ocorrendo em etapas, de forma semelhante a uma propagação por fadiga. Observa-se também que as amostras apresentam certa descamação (semelhante à clivagem).

Outra técnica que os autores utilizam para avaliar os efeitos do stress cracking é a relaxação de tensão ${ }^{[18,27,28]}$. Neste procedimento, o corpo de prova é tensionado até uma determinada força e é mantido em deformação constante, sendo possível determinar a taxa de relaxação, definida como o decaimento da força aplicada com o tempo.

Quando a análise de relaxação de tensão é feita na presença do agente de stress cracking, ocorre liberação de energia devido à plastificação localizada, com formação e propagação de crazes, o que reduz a força aplicada sob deformação constante ${ }^{[29]}$. Assim, a taxa de relaxação pode ser relacionada à falha por ESC, sendo dependente da quantidade e velocidade de propagação das trincas formadas. O que comumente se tem observado nos ensaios de ESC sob relaxação de tensão é que maiores graus de fissuramento e menores velocidades de propagação das trincas proporcionam maiores taxas de relaxaçã ${ }^{[18,27,28]}$. $\mathrm{Na}$ Tabela 1 tem-se as taxas de relaxação dos materiais em contato com concentrações de 0,25 e $1 \mathrm{M}$ de $\mathrm{NaOH}$, sob as forças iniciais de 1300 e $1600 \mathrm{~N}$. Observa-se que a taxa de relaxação aumenta com a força e com a concentração de $\mathrm{NaOH}$ para todos os materiais estudados. Para a força de $1600 \mathrm{~N}$, analisando individualmente cada concentração, os materiais exibem relaxação semelhante (considerando os desvios), sugerindo que em tensões elevadas o efeito da velocidade rápida de propagação da trinca é mais significativo que o da morfologia do material. Em tensões mais baixas, os híbridos PET/argila exibem maiores taxas de relaxação que o PET puro, sendo discretamente maior para o hibrido $\mathrm{H} 1-1 \%$. Isso sugere que a argila na forma agregada e ordenada favorece uma maior concentração de tensão, o que causa uma maior deformação plástica local à frente da trinca durante a propagação, resultando em relaxações maiores, mesmo que a quantidade de trincas formadas seja menor (Figura 4c). Já a argila com empilhamento de lamelas mais desordenado possivelmente favoreça uma menor concentração de tensão, com consequente redução na velocidade de propagação das trincas, induzindo a nucleação de mais trincas. Isso explicaria o fato do híbrido $\mathrm{H} 2-1 \%$ fraturar com maior grau de fissuramento superficial (conforme pode ser visualizado na Figura 4) em comparação com o H1-1\%, mesmo nos casos que apresentam relaxação semelhante.

Tabela 1. Taxas de relaxação dos materiais testados nas forças de 1300 e $1600 \mathrm{~N}$ e em contato com soluções aquosas de $\mathrm{NaOH}$ nas concentrações de 0,25 e $1 \mathrm{M}$.

\begin{tabular}{ccccc}
\hline \multirow{2}{*}{ Força (N) } & \multirow{2}{*}{ Concentração } & \multicolumn{3}{c}{ Taxa de Relaxação (N/s) } \\
\cline { 3 - 5 } & & PET & H1-1\% & H2-1\% \\
\hline \multirow{2}{*}{1300} & $0,25 \mathrm{M}$ & $1,148 \pm 0,015$ & $1,861 \pm 0,175$ & $1,383 \pm 0,140$ \\
& $1 \mathrm{M}$ & $2,584 \pm 0,213$ & $3,211 \pm 0,433$ & $3,366 \pm 0,115$ \\
\multirow{2}{*}{1600} & $0,25 \mathrm{M}$ & $3,092 \pm 0,702$ & $2,246 \pm 0,022$ & $2,587 \pm 0,237$ \\
& $1 \mathrm{M}$ & $4,104 \pm 0,115$ & $4,371 \pm 0,038$ & $4,368 \pm 0,096$ \\
\hline
\end{tabular}

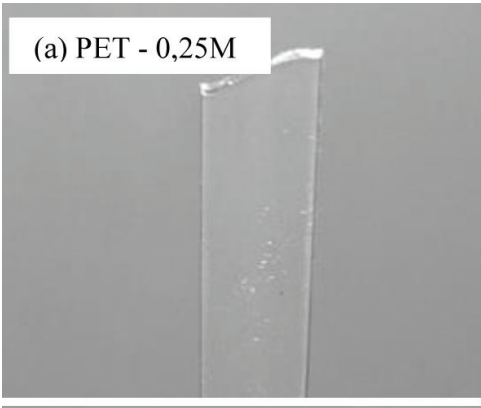

(b) PET - 1M
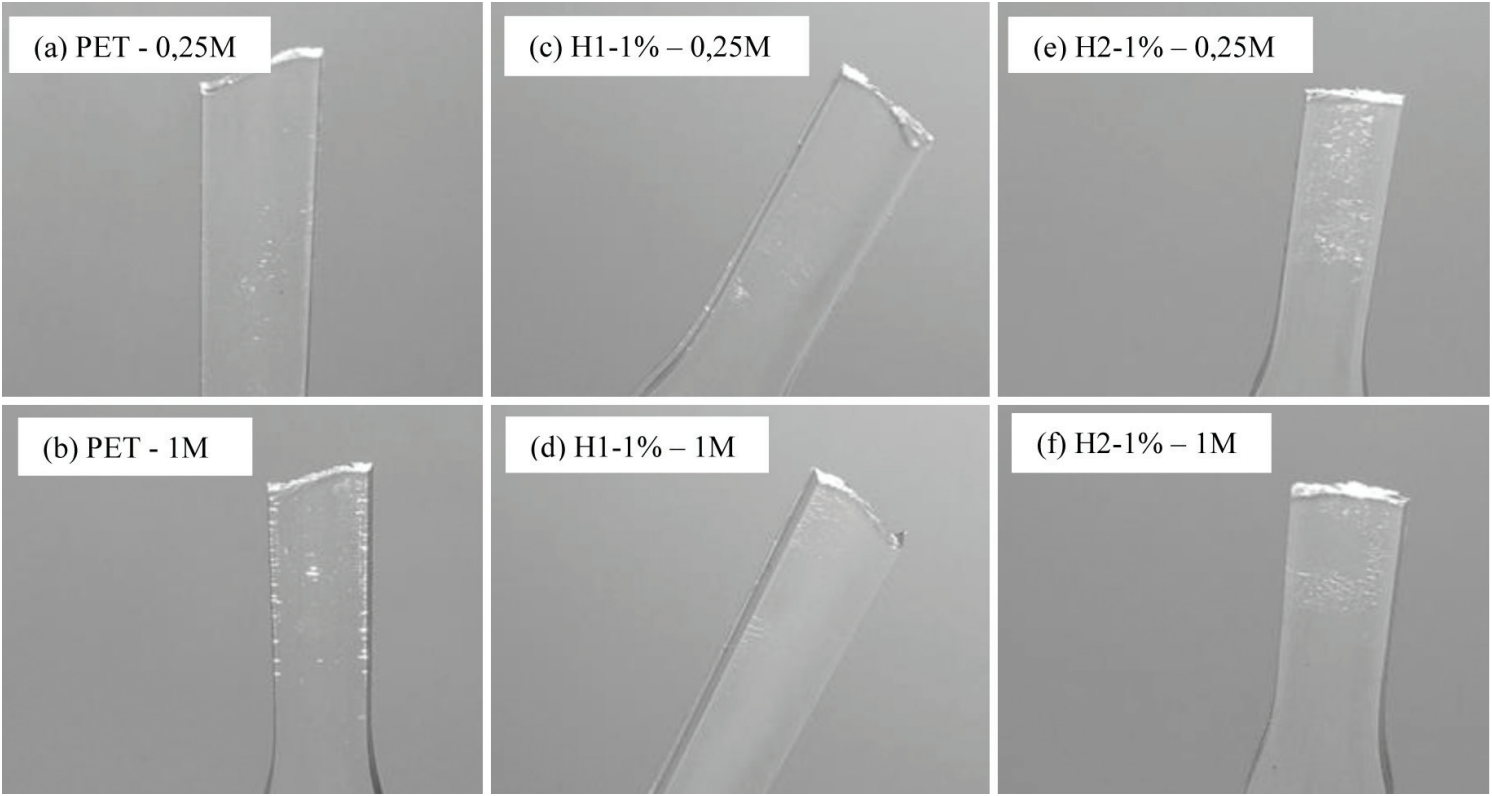

(f) $\mathrm{H} 2-1 \%-1 \mathrm{M}$

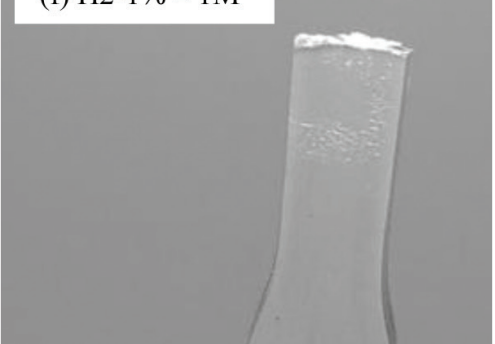

Figura 4. Fotografias de corpos de prova fraturados durante o ensaio de relaxação na força inicial de $1600 \mathrm{~N}$ e em contato com soluções aquosas de $\mathrm{NaOH}$ nas concentrações de 0,25 e $1 \mathrm{M}$. 


\section{Influência do teor de carga}

A análise da influencia do teor de carga na falha por ESC foi realizada com os híbridos obtidos com a argila seca a $110^{\circ} \mathrm{C}$ por $24 \mathrm{~h}$, visto essa condição de secagem ter favorecido uma melhor interação carga/matriz (conforme anteriormente observado para os híbridos $\mathrm{H} 1-1 \%$ e H2-1\%). As propriedades mecânicas dos materiais com diferentes teores de carga podem ser comparadas na Figura 5. O aumento no teor de carga praticamente não alterou a resistência do PET, pois os valores obtidos com 3\% e 5\% são semelhantes ao de 1\% (Figura 5a) e, assim sendo, representam uma diminuição de cerca de $10 \%$ na resistência do material, como mostrado anteriormente (Figura 2). A resistência ao ESC sob tração também não é muito afetada pelo teor de carga. Considerando o desvio-padrão, a diminuição da resistência tênsil pela presença de argila foi em torno de $50 \%$. Por outro lado, a ductilidade do material, como era de se esperar, diminui com o teor de carga (Figura 5b).

Os resultados para a taxa de relaxação na presença de diferentes concentrações de solução aquosa de $\mathrm{NaOH}$ estão mostrados na Tabela 2. Observa-se que a taxa de relaxação aumenta com a força aplicada e com a concentração de $\mathrm{NaOH}$ para os híbridos estudados quando na presença das concentrações de 0,25 e $1 \mathrm{M}$. Tal efeito era o esperado, uma vez que a ação conjunta da tensão mecânica e fluido agressivo ocasiona a formação e propagação de crazes e nesse processo ocorre liberação de energia, devido à plastificação localizada, reduzindo a força aplicada sob deformação constante, ou seja, aumentando a taxa de relaxação. Observa-se também que na presença das concentrações de 0,25 e 1M, os híbridos com maiores teores de argila apresentam maiores taxas de relaxação, sugerindo que as partículas de argila podem estar concentrando tensão. Essa concentração de tensão, obviamente, é intensificada quando maiores teores de carga estão presentes. Tal suposição é reforçada ao se analisar os fissuramentos provocados nos corpos de prova (Figura 6). Como a taxa de relaxação está relacionada com as quantidades de trincas formadas e são visualizadas poucas fissuras nos corpos de prova fraturados (Figura 6c-f), o que justificaria a maior relaxação seria a concentração de tensão, uma vez que maiores concentrações de tensão causaria maior deformação plástica local à frente da trinca durante a propagação, resultando em maiores relaxações. Com a concentração de $3 \mathrm{M}$, apesar dos altos desvios, os híbridos com maiores teores de argila apresentam menor relaxação, e esta não é maximizada com a tensão, sugerindo um possível efeito de barreira ao fluido.

\section{Influência na degradação do polímero}

Com o objetivo de verificar a influencia da argila no processo de absorção de solução pelo PET como também em sua degradação sob longos períodos de exposição às soluções causadoras de ESC, foram realizadas medidas de variação de massa em corpos de prova de PET e de híbridos

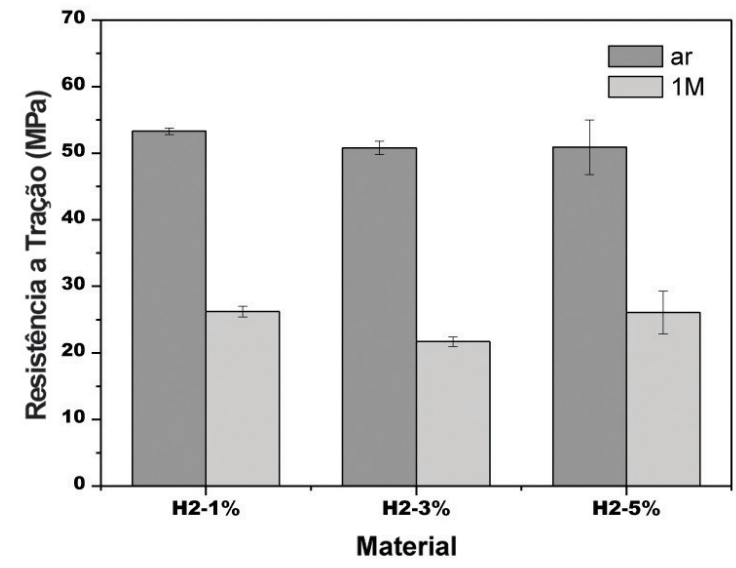

(a)

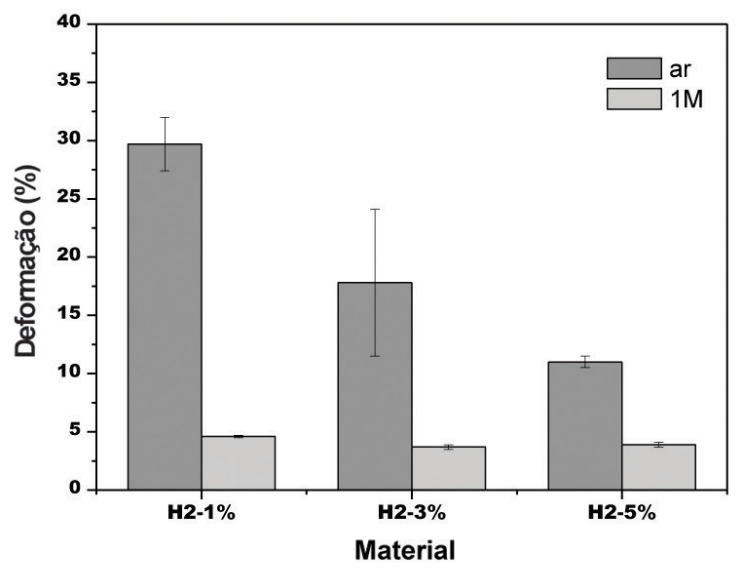

(b)

Figura 5. Propriedades mecânicas de híbridos com 1,3 e 5\% de argila na ausência (ar) e na presença de solução aquosa de $\mathrm{NaOH}$ na concentração de $1 \mathrm{M}$.

Tabela 2. Taxas de relaxação dos híbridos com teores de 1, 3 e 5\% de argila, testados em diferentes forças e concentrações de solução aquosa de hidróxido de sódio.

\begin{tabular}{ccccc}
\hline \multirow{2}{*}{ Força (N) } & \multirow{2}{*}{ Concentração } & \multicolumn{3}{c}{ Taxa de Relaxação (N/s) } \\
\cline { 3 - 5 } & & H2-1\% & H2-3\% & H2-5\% \\
\hline \multirow{2}{*}{1300} & $0,25 \mathrm{M}$ & $1,383 \pm 0,140$ & $2,185 \pm 0,206$ & $1,817 \pm 0,174$ \\
& $1 \mathrm{M}$ & $3,366 \pm 0,115$ & $3,424 \pm 0,938$ & $3,941 \pm 0,372$ \\
& $3 \mathrm{M}$ & $9,884 \pm 0,421$ & $6,539 \pm 1,498$ & $7,338 \pm 3,421$ \\
\multirow{2}{*}{1600} & $0,25 \mathrm{M}$ & $2,587 \pm 0,237$ & $3,019 \pm 0,531$ & $3,343 \pm 0,269$ \\
& $1 \mathrm{M}$ & $4,368 \pm 0,096$ & $4,908 \pm 0,224$ & $5,949 \pm 0,529$ \\
& $3 \mathrm{M}$ & $8,105 \pm 1,536$ & $4,664 \pm 0,396$ & $5,934 \pm 1,714$ \\
\hline
\end{tabular}


com $1 \%$ de argila, em função do tempo de submersão em diferentes concentrações de solução aquosa de $\mathrm{NaOH}$. Sabe-se que um material polimérico em contato com fluidos pode sofrer alterações como: ganho de massa (caso o polímero absorva o fluido), perda de massa (se o polímero for degradado pelo fluido ou se este extrai moléculas de baixa massa molar do polímero), dissolução (se o fluido for um bom solvente) ou outras alterações, tais como perda de transparência e variações de cor ${ }^{[30]}$.

Os resultados da variação de massa em função do tempo de submersão nas soluções com concentrações de 1 e 3M estão mostrados na Figura 7. Observa-se que após o primeiro dia de submersão já começa a ocorrer perda de massa pelas amostras e que o híbrido apresenta perda consideravelmente maior que o PET puro indicando que a presença de argila pode estar acelerando o processo degradativo do polímero em contato com fluidos agressivos.

Mesmo na ausência de fluidos agressivos, há relatos de que cargas argilosas aceleram a degradação da matriz de PET $^{[31-33]}$. Essa ação degradativa é atribuída aos grupos hidroxila presentes nas plaquetas de argila, os quais seriam responsáveis por provocar cisão hidrolítica de ligações éster nas moléculas de PET ${ }^{[33]}$. De acordo com Bär ${ }^{[34]}$, os sítios catalizadores de degradação presentes na argila podem ser decorrentes tanto dos grupos hidroxila, os quais são fortemente ácidos no vértice da camada do silicato, como de íons de metais de transição que não foram trocados no interior das galerias ou de defeitos cristalográficos entre as camadas.
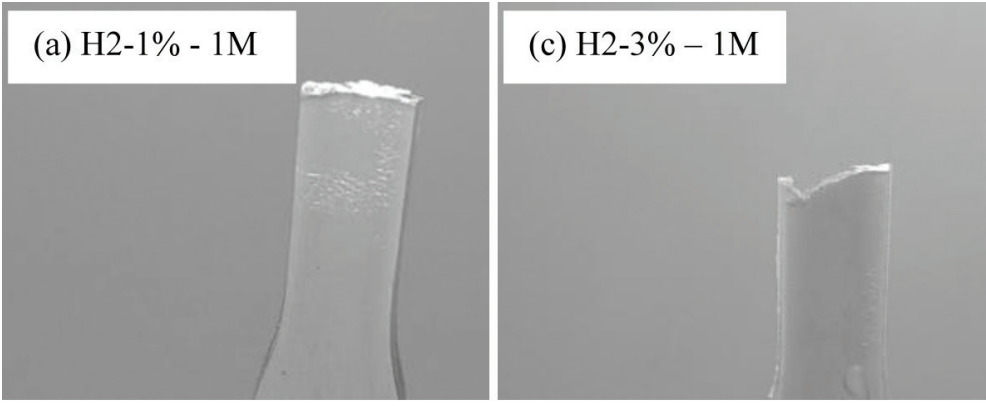

(e) $\mathrm{H} 2-5 \%-1 \mathrm{M}$

(b) $\mathrm{H} 2-1 \%-3 \mathrm{M}$

(d) $\mathrm{H} 2-3 \%-3 \mathrm{M}$
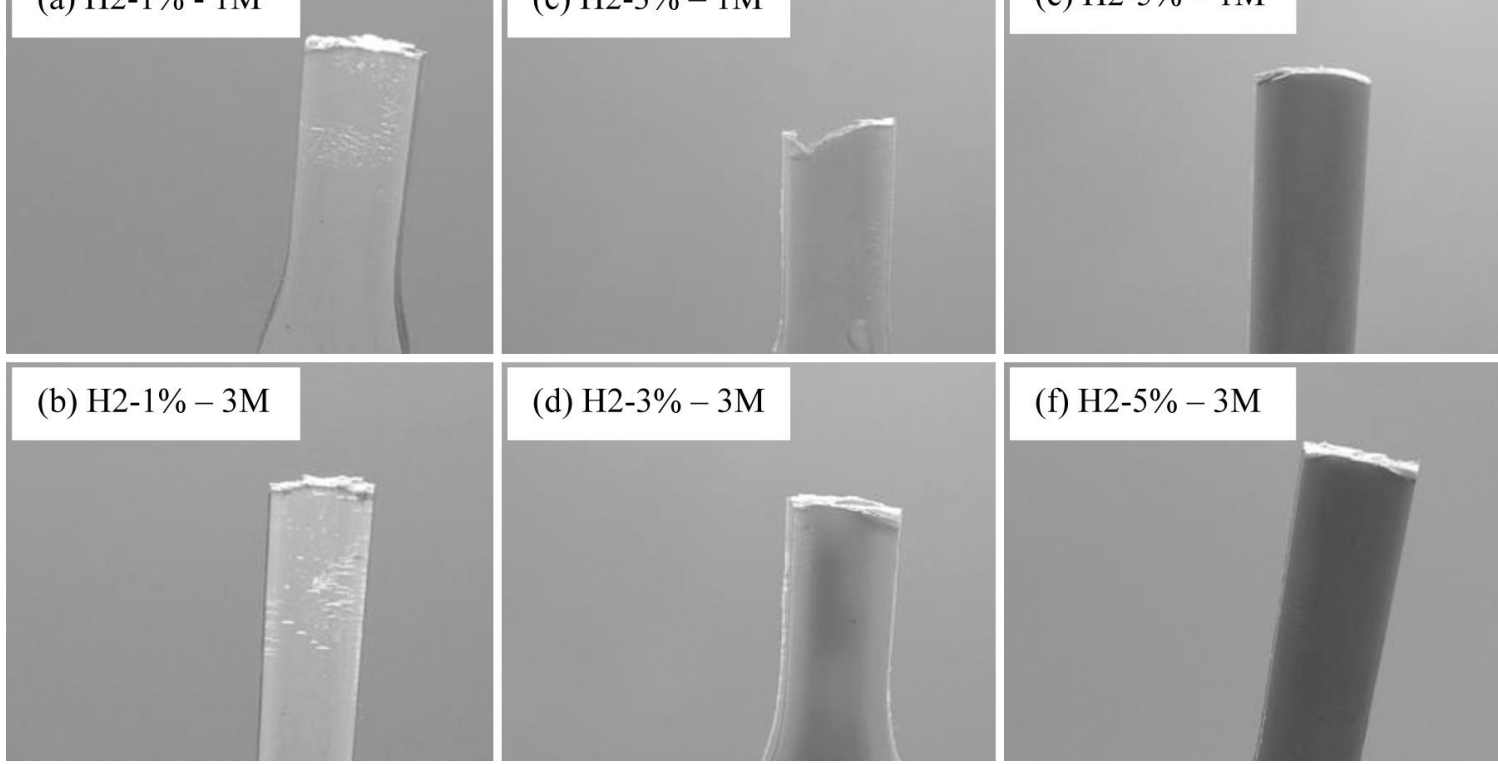

(f) $\mathrm{H} 2-5 \%-3 \mathrm{M}$

Figura 6. Comparação entre os fissuramentos apresentados pelos híbridos com 1, 3 e 5\% de argila na força de $1600 \mathrm{~N}$ e sob soluções aquosas de $\mathrm{NaOH}$ nas concentrações de 1 e $3 \mathrm{M}$.

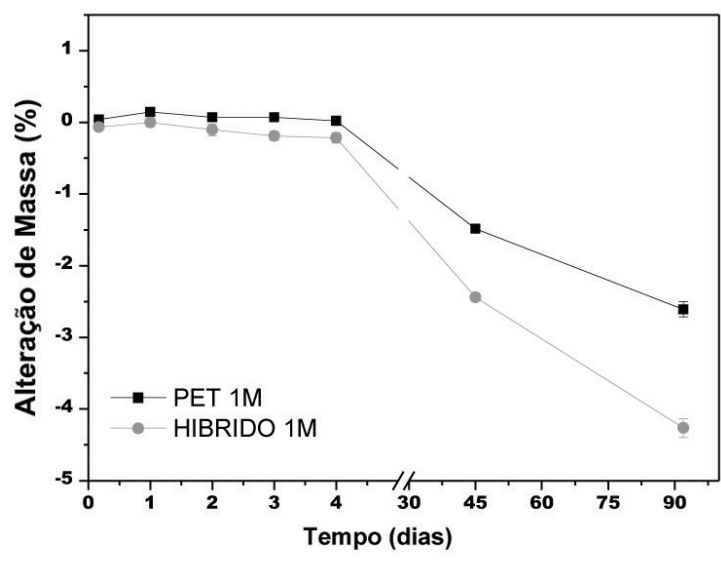

(a)

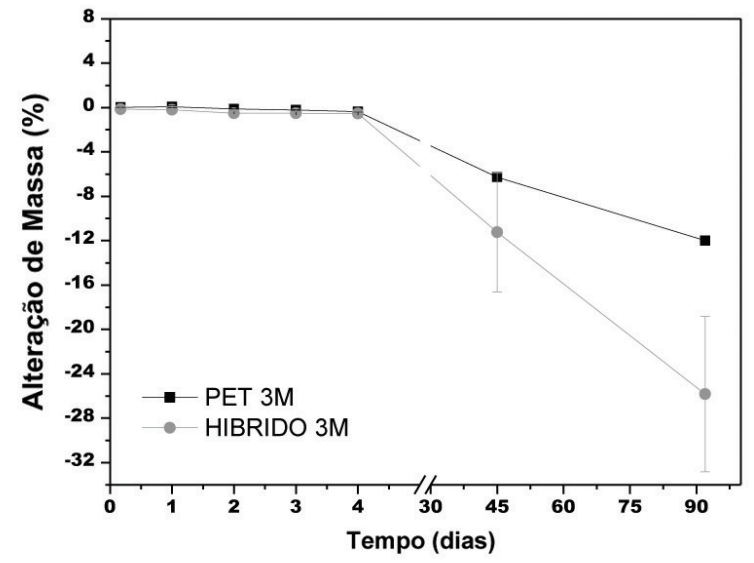

(b)

Figura 7. Comparação entre a variação de massa do PET e do híbrido com 1\% de argila em soluções aquosas de $\mathrm{NaOH}$. 
Tabela 3. Massa molar ponderal média do híbrido com $1 \%$ de argila submetido ao ensaio de relaxação de tensão em diferentes forças e em contato com solução aquosa de $\mathrm{NaOH}$ nas concentrações de 1 e $3 \mathrm{M}$

\begin{tabular}{ccc}
\hline \multirow{2}{*}{ Força (N) } & \multicolumn{2}{c}{$\mathbf{M}_{\mathbf{w}}$} \\
\cline { 2 - 3 } & $\mathbf{1 M}$ & $\mathbf{3 M}$ \\
\hline 1300 & $27.450 \pm 141$ & $16.070 \pm 257$ \\
1600 & $27.710 \pm 158$ & $16.350 \pm 170$ \\
\hline Húbrido PET/argila na auência de soluções: $\mathrm{M}_{\mathrm{w}}=28.410 \pm 176$.
\end{tabular}

Híbrido PET/argila na ausência de soluções: $\mathrm{M}_{w}=28.410 \pm 176$.

Tabela 4. Influência da força aplicada no ensaio de relaxação de tensão na degradação do PET e do híbrido com 1\% de argila em contato com solução aquosa de $\mathrm{NaOH}$ na concentração de $1 \mathrm{M}$.

\begin{tabular}{ccc}
\hline \multirow{2}{*}{ Material } & \multicolumn{2}{c}{$\mathbf{M}_{\mathrm{w}}$} \\
\cline { 2 - 3 } & $\mathbf{1 3 0 0 N}$ & $\mathbf{1 6 0 0 N}$ \\
\hline PET & $30.340 \pm 293$ & $25.970 \pm 189$ \\
PET/argila & $27.450 \pm 141$ & $27.710 \pm 158$ \\
\hline
\end{tabular}

Tabela 5. Influência da concentração da solução aquosa de $\mathrm{NaOH}$ na degradação do PET e do híbrido com $1 \%$ de argila durante o ensaio de relaxação de tensão na força de $1600 \mathrm{~N}$.

\begin{tabular}{ccc}
\hline \multirow{2}{*}{ Material } & \multicolumn{2}{c}{$\mathbf{M}_{\mathbf{w}}$} \\
\cline { 2 - 3 } & $\mathbf{1 M}$ & $\mathbf{3 M}$ \\
\hline PET & $25.970 \pm 189$ & $17.640 \pm 110$ \\
PET/argila & $27.710 \pm 158$ & $16.350 \pm 170$ \\
\hline
\end{tabular}

Já foi verificado que durante os ensaios de ESC as soluções aquosas de hidróxido de sódio atacam quimicamente o PET, reduzindo significativamente sua massa molar ${ }^{[27,28]}$ e que a quantidade e concentração da solução e a tensão mecânica exercem considerável influência nesse processo degradativo ${ }^{[18]}$. Anteriormente foi mostrado que a degradação depende do tempo de exposição e ficou evidenciado que pode ser favorecida pela presença de argila (Figura 7). Para verificar a influencia da argila na degradação do polímero no pouco tempo de exposição às soluções durante os ensaios de ESC, foram realizadas medidas de viscosidade para determinação da massa molar do híbrido PET/argila após os ensaios conduzidos sob relaxação. Os resultados mostrados na Tabela 3 indicam que, diferentemente do PET puro, a massa molar apresenta uma variação maior com a concentração de $\mathrm{NaOH}$ e pouca dependência com a força aplicada. Na presença de soluções a $1 \mathrm{M}$ ocorre uma diminuição de apenas 3\% na massa molar do polímero, sugerindo que a presença de argila, de certa forma, impede um ataque químico mais pronunciado com essa concentração (possivelmente por alterar a interação fluido-polímero). Já em concentrações mais elevadas o efeito é de pronunciamento do ataque químico, pois quando se utiliza a concentração de $3 \mathrm{M}$ a massa molar diminui drasticamente - cerca de $43 \%$.

As Tabelas 4 e 5 mostram as massas molares do PET puro e do híbrido com $1 \%$ de argila avaliando a influencia da força aplicada e da concentração de $\mathrm{NaOH}$, respectivamente. Observa-se que para o híbrido PET/argila não ocorrem alterações significativas com o aumento da tensão mecânica, ao passo que para o PET puro o aumento na tensão ocasiona um incremento de aproximadamente $13 \%$ no ataque químico (Tabela 4). East \& $\operatorname{Rahman}^{[35]}$ também destacaram a influência da tensão mecânica na degradação de polímeros ao observar que a hidrólise do PET foi acelerada pela aplicação de tensão. Segundo os autores a presença de grupos carboxila e hidroxila contribuem para a hidrofilicidade da superfície do PET.

Em relação à concentração da solução, observa-se que ambos os materiais são fortemente influenciados, sendo essa influência ainda mais significativa para o híbrido PET/ argila. Para o PET puro o aumento na concentração favorece um incremento de $32 \%$ na degradação, ao passo que para o híbrido o incremento é de $41 \%$ (Tabela 5), indicando que a presença de argila favorece o processo degradativo sob elevadas concentrações de solução alcalina. Quanto maior a concentração da solução, maior a quantidade de grupos hidroxila que se somam aos já existentes nas plaquetas da argila para favorecer o ataque químico da matriz polimérica, uma vez que esses grupos são considerados catalisadores da degradação do $\mathrm{PET}^{[31,32,34,35]}$.

\section{Conclusões}

A presença de argila diminui drasticamente a ductilidade do polímero e essa diminuição é função da morfologia e do teor de carga. A concentração de tensão pela carga também é dependente desses fatores. Maiores teores de carga e empilhamento mais regular das lamelas de argila promovem maiores concentrações de tensão, favorecendo a rápida propagação de trincas e, consequentemente, menores fissuramentos superficiais. Por outro lado, um empilhamento mais desordenado das lamelas de argila provoca menores concentrações de tensão, porém favorece a nucleação de mais trincas, sugerindo que o efeito de barreira ao fluido não é efetivo e que os danos por stress cracking em polímeros contendo argila dependem principalmente da concentração de tensão ocasionada pela carga. A presença de argila também afeta o ataque químico que ocorre simultaneamente ao ESC do PET em soluções alcalinas. Em baixas concentrações o ataque químico é menos pronunciado para o PET com argila, ao passo que em elevadas concentrações a argila favorece um maior ataque químico, mesmo que o efeito da tensão mecânica durante o processo degradativo seja minimizado.

\section{Agradecimentos}

Os autores agradecem ao CNPq pelo auxílio financeiro e a $M \& G$ pela doação do polímero.

\section{Referências Bibliográficas}

1. Romão, W.; Spinacé, M. A. S. \& Paoli, M.-A. D. - Polímeros., 19, p.121 (2009). http://dx.doi.org/10.1590/S010414282009000200009

2. Wiebeck, H. \& Harada, J. - "Plásticos de engenharia: tecnologia e aplicações". Artiliber, São Paulo (2005).

3. Wang, Y.; Gao, J.; Ma, Y. \& Agarwal, U. S. - Compos., Part B Eng., 37, p.399 (2006). http://dx.doi.org/10.1016/j. compositesb.2006.02.014.

4. Sánchez-Solís, A.; Romero-Ibarra, I.; Estrada, M. R.; Calderas, F. \& Manero, O. - Polym. Eng. Sci., 44, p.1094 (2004). http:// dx.doi.org/10.1002/pen.20102. 
5. Wan, T.; Chen, L.; Chua, Y. C. \& Lu, X. - J. Appl. Polym. Sci., 94, p.1381 (2004). http://dx.doi.org/10.1002/app.20975.

6. Ou, C. F.; Ho, M. T. \& Lin, J. R. - J. Appl. Polym. Sci., 91, p.140 (2004). http://dx.doi.org/10.1002/app.13158.

7. Davis, C. H.; Mathias, L. J.; Gilman, J. W.; Schiraldi, D. A.; Shields, J. R.; Trulove, P.; Sutto, T. E. \& Delong, H. C. - J. Polym. Sci., B, Polym. Phys., 40, p.2661 (2002). http://dx.doi. org/10.1002/polb.10331.

8. Ezrin, M. - "Plastics failure guide", Hanser, Munich (1996).

9. Lustiger, A. Medical Plastics and Biomaterials Magazine, p.66, July (1996).

10. Jansen, J. A. Adv. Mater. Process., p.50, June (2004).

11. Turnbull,A.; Maxwell,A. S. \& Pillai, S. - Polym. Test., 19, p.117 (2000). http://dx.doi.org/10.1016/S0142-9418(98)00076-2.

12. Hansen, C. M. - Polym. Degrad. Stabil., 77, p.43 (2002). http:// dx.doi.org/10.1016/S0141-3910(02)00078-2.

13. Nielsen, T. B. \& Hansen, C. M. - Polym. Degrad. Stabil., 89, p.513 (2005). http://dx.doi.org/10.1016/j. polymdegradstab.2004.12.027.

14. Kjellander, C. K.; Nielsen, T. B.; Ghanbari-Siahkali, A.; Kingshott, P.; Hansen, C. M. \& Almdal, K. - Polym. Degrad. Stabil., 93, p.1486 (2008). http://dx.doi.org/10.1016/j. polymdegradstab.2008.05.011.

15. Paul, D. R. \& Robeson, L. M. - Polymer., 49, p.3187 (2008).

16. Sinha Ray, S. \& Okamoto, M. - Prog. Polym. Sci., 28, p.1539 (2003). http://dx.doi.org/10.1016/j.progpolymsci.2003.08.002.

17. Calcagno, C. I. W. - "Estudo da morfologia, do comportamento de cristalização e das propriedades mecânicas de nanocompósitos de PET e PP/PET com montmorilonita", Tese de Doutorado, Universidade Federal do Rio Grande do Sul, Brasil (2007).

18. Teófilo, E. T. \& Rabello, M. S. - REMAP, 8, p.141 (2013).

19. Berkowitz, S. A. - J. Appl. Polym. Sci., 29, p.4353 (1984). http://dx.doi.org/10.1002/app.1984.070291264.

20. Ghanbari, A.; Heuzey, M. C.; Carreau, P. J. \& Ton-That, M. T. - Polymer (Guildf.)., 54, p.1361 (2013). http://dx.doi. org/10.1016/j.polymer.2012.12.066.

21. Beatrice, C. A. G. - "Estudo das propriedades reológicas de nanocompósitos para a produção de filmes tubulares", Dissertação de Mestrado, Universidade Federal de São Carlos, Brasil (2008).
22. Calcagno, C. I. W.; Mariani, C. M.; Teixeira, S. R. \& Mauler, R. S. - Polymer (Guildf.)., 48, p.966 (2007). http://dx.doi. org/10.1016/j.polymer.2006.12.044.

23. Barber, G. D.; Calhoun, B. H. \& Moore, R. B. - Polymer (Guildf.)., 46, p.6706 (2005). http://dx.doi.org/10.1016/j. polymer.2005.05.024.

24. Costache, M. C.; Heidecker, M. J.; Manias, E. \& Wilkie, C. A. - Polym. Adv. Technol., 17, p.764 (2006). http://dx.doi. org/10.1002/pat.752.

25. Leite, I. F.; Raposo, C. M. O.; Malta, O. M. L.; Silva, S. M. L. REMAP, 5, p.5 (2010)

26. Carastan, D. J. - "Obtenção e caracterização reológica de nanocompósitos de polímeros estirênicos", Tese de Doutorado, Universidade de São Paulo, Brasil (2007).

27. Teófilo, E. T.; Melo, R. N.; Silva, S. M. L. \& Rabello, M. S. - Polímeros., 19, p.202 (2009). http://dx.doi.org/10.1590/ S0104-14282009000300008.

28. Teófilo, E. T.; Silva, S. M. L. \& Rabello, M. S. - J. Appl. Polym. Sci., 118, p.3089 (2010). http://dx.doi.org/10.1002/ app. 32748 .

29. Sousa, A. R.; Araujo, E. S.; Carvalho, A. L.; Rabello, M. S. \& White, J. R. - Polym. Degrad. Stabil., 92, p.1465 (2007). http://dx.doi.org/10.1016/j.polymdegradstab.2007.05.008.

30. Moskala, E. J.; Jones, M. Medical Plastics and Biomaterial Magazine, p.34, May (1998).

31. Xu, X.; Ding, Y.; Qian, Z.; Wang, F.; Wen, B.; Zhou, H.; Zhang, S. \& Yang, M. - Polym. Degrad. Stabil., 94, p.113 (2009). http://dx.doi.org/10.1016/j.polymdegradstab.2008.09.009.

32. Bikiaris, D. - Thermochim. Acta., 523, p.25 (2011). http:// dx.doi.org/10.1016/j.tca.2011.06.012.

33. Donelli, I.; Freddi, G.; Nierstrasz, V. A. \& Taddei, P. - Polym. Degrad. Stabil., 95, p.1542 (2010). http://dx.doi.org/10.1016/j. polymdegradstab.2010.06.011.

34. Bär, M. - "Estudo da fotooxidação de nanocompósito PP/ bentonita", Dissertação de Mestrado, Universidade Estadual de Ponta Grossa, Brasil (2008).

35. East, G. C. \& Rahman, M. - Polymer (Guildf.)., 40, p.2281 (1999). http://dx.doi.org/10.1016/S0032-3861(98)00451-0.

Enviado: Dez. 06, 2013

Reenviado: Mar. 21, 2014 Aceito: Maio 15, 2014 